\title{
Rupture of the cavernous body diagnosed by contrast-enhanced ultrasound: Presentation of a clinical case
}

\author{
Lucio Dell'Atti $^{1}$, Simone Scarcella ${ }^{1}$, Giulio Argalia ${ }^{2}$, Lorenzo Montesi ${ }^{1}$, Gian Marco Giuseppetti ${ }^{2}$, \\ Andrea Benedetto Galosi ${ }^{1}$ \\ ${ }^{1}$ Department of Urology, Polytechnic University of Marche Region, University Hospital "Ospedali Riuniti”, Ancona, Italy; \\ ${ }^{2}$ Department of Radiology, Polytechnic University of Marche Region, University Hospital "Ospedali Riuniti”, Ancona, Italy.
}

\begin{abstract}
Summary Penile trauma represents a urological emergency characterized by the breaking of the albuginea tunic. A fast diagnosis and early surgical repair are the best treatments to avoid post-operative sequelae such as curvatures or deformities of the penis. An ultrasound evaluation may not be able to identify the injury in the tunica albuginea due to the edematous swelling of the penis and clots within the tear deteriorate the image contrast and can hide the injury. We here report a case study of successful management via surgical treatment for rupture of the cavernous body diagnosed by contrast-enhanced ultrasound in a young patient with penile trauma.
\end{abstract}

KEY WORDS: Penile fracture; Trauma; Ultrasound; Contrast enhanced ultrasonography.

Submitted 2 April 2018; Accepted 29 April 2018

\section{INTRODUCTION}

The fracture of the penis represents an urological emergency characterized by the breaking of the tunica albuginea and cavernous body (CB), frequently resulting in direct trauma, vigorous sexual intercourse, falls, forceful manipulation and masturbation (1). The diagnosis is usually delayed because of the patient's embarrassment.

A fast diagnosis and early surgical repair are the best treatments to avoid post-operative sequelae such as curvatures or deformities of the penis (2). We here report a case study of successful management via surgical treatment for rupture of the $\mathrm{CB}$ diagnosed by contrast-enhanced ultrasound (CEUS) in a patient with penile trauma.

\section{Case Report}

A 25-years-old male patient presented to our Emergency Department for severe penile pain and suspicion of penile fracture after trauma during an act of masturbation. On presentation, the penis showed swelling, ecchymosis without deformity and urethrorrhagia. An ultrasonographic evaluation (US), 7-12 MHz multi-frequency linear probe, was performed first on a transversal plane, from the glans to the base of the penile shaft, and then completed with a longitudinal scan. US examination of the penis reported a hypo-anechoic mass on the right $\mathrm{CB}$, highly suspicious for hematoma but without certain interruption of the tunica albuginea (Figure 1A, 1B).

Due to difficulty detecting the cause of acute hematoma, a CEUS study was performed through insertion of a 19 Gauge butterfly needle in the corpora cavernosa. CEUS examination showed a tunica albuginea's defect in the right $\mathrm{CB}$ with a concomitant area characterized by absence of contrast enhancement, described as compatible with hematoma (Figure 1C). An emergency explorative surgery was immediately performed. After circumcision and degloving of the penile shaft we evacuated the hematoma and individuated a tear (maximum diameter $8 \mathrm{~mm}$ ) in the right $\mathrm{CB}$ tunica albuginea. A running suture with absorbable 2-0 polydioxanone, was used for tear repair. No postoperative complications occurred and after 3 days the patient was discharged.

\section{Discussion}

The use of US in traumas is now accepted in clinical practice as initial diagnostic evaluation of blunt abdominal trauma. However, up to date, ultrasonographic study of parenchymal lesions is characterized by the presence of frequent false negatives (3). Penile traumas generally occur in the proximal or mid shaft of the erect penis, and concomitant lesions to the urethra occur in approximately $10-20 \%$ of patients $(1,2)$. Penile fractures are urologic emergencies and require as soon as possible surgical repair. The diagnosis of penile fracture is usually established based on its characteristic history and physical findings (1).

However, in all cases an ultrasonographic evaluation should be performed to define the localization of the lesion $(2,3)$. It is important considering that subcutaneous hematoma confined to the Buck's fascia, in absence of a tunica albuginea laceration, requires medical conservative treatment. Only if a tunica albuginea tear is suspected or confirmed by US is mandatory to perform surgery within 24 from the traumatic event in order to reduce hospitalization, perioperative penile deviation and erectile dysfunction risk (2). The echo structure of the corpora cavernosa are homogeneous and 


\section{Figure 1.}

(A): Longitudinal and (B): transverse ultrasound image show an injury of the dorsal subtunical venous plexus in the absence of complete tunica disruption (white arrow).

(C): Late phase transverse CEUS dual mode showing the avascular area representing hematoma and the tear of the right cavernous body as an interruption of the thin echogenic line of the tunica albuginea (white arrow).

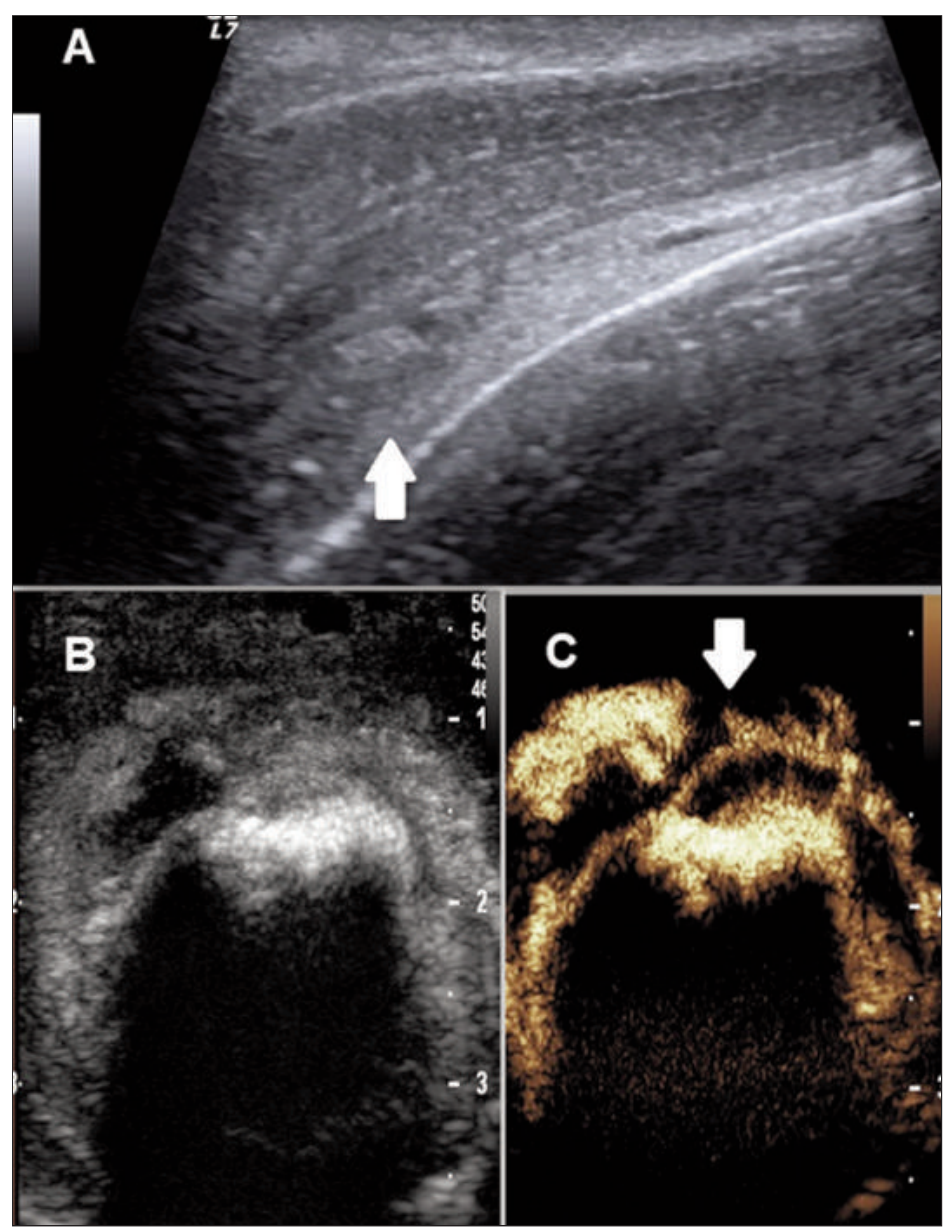

CEUS can improve tissue delineation and a better evaluation of the tunica albuginea integrity. MRI, however, it is expensive and rarely used in the evaluation of acute penile trauma $(1,4)$

CEUS, using micro-bubbles contrast agents and complementary harmonic pulse sequences can implements the diagnostic performance of the Bmode, distinguish the parenchymal perfusion and defects. Moreover, it improves patient tolerance, reduces radiation exposure and can be also safely performed in patients with renal impairment $(4,5)$. Guidelines and Recommendations on Clinical Practice of CEUS represent current indications for administration of ultrasound contrast enhancement agents in different urogenital pathologies, including penile conditions (6).

Conservative management of penile trauma lead to increased rate of complications, such as penile curvature or erectile dysfunction, compared to immediate surgical intervention. CEUS is noninvasive, widely viable and may help in the process of exact localization of the tear site in the tunica albuginea, especially in complex and atypical cases.

\section{References}

1. Nomura JT, Sierzenski PR. Ultrasound diagnosis of penile fracture. J Emerg Med. 2010; 38:362-365.

2. Dell'Atti L. The role of ultrasonography in the diagnosis and management of penile trauma. J Ultrasound. 2016; 19:161-166.

3. Topsøe JF, Dencker D. Investigation of Penile Conditions by Ultrasound and Contrast-Enhanced Ultrasound Presentation of Three Clinical Cases. Ultrasound Int Open. 2015; 1:E78-79.

4. Cokkinos DD, Antypa E, Kalogeropoulos I, et al. Contrast-enhanced ultrasound performed under urgent conditions. Indications, review of the technique, clinical examples and limitations. Insights Imaging. 2013; 4:185-198. identified as two hypoechoic circular structures surrounded by a thin hyperechoic line representing the tunica albuginea (4). In case of edematous swelling and massive clots formation close to the tunica defect, an only US in B-mode could be misleading (2). For a better characterization Magnetic Resonance Imaging (MRI) or
5. Catalano O, Aiani L, Barozzi L, et al. CEUS in abdominal trauma: multi-center study. Abdom Imaging. 2009; 34:225-234.

6. Tranquart F, Mercier L, Frinking P, et al. Perfusion quantification in contrast-enhanced ultrasound (CEUS)--ready for research projects and routine clinical use. Ultraschall Med. 2012; (33 Suppl1):S31-8.

\section{Correspondence}

Lucio Dell'Atti, MD, PhD

dellatti@hotmail.com

Simone Scarcella, MD

Lorenzo Montesi, MD

Andrea Benedetto Galosi, MD

Department of Urology, Polytechnic University of Marche Region,

University Hospital "Ospedali Riuniti"

via Conca 71, 60126 Ancona, Italy

Giulio Argalia, MD

Gian Marco Giuseppetti, MD

Department of Radiology, Polytechnic University of Marche Region,

University Hospital "Ospedali Riuniti", Ancona, Italy 